\title{
Mars as a Competitive Candidate for Inclusion in the New Frontiers Mission List: MEPAG Community Perspectives
}

\section{Prepared on behalf of the Mars Exploration Program Analysis Group (MEPAG) \\ by the MEPAG Steering Committee as Input to the 2023-2032 Planetary Science and Astrobiology Decadal Survey}

Contact information: Dr. R. Aileen Yingst, MEPAG chair, Planetary Science Institute; 920-360-3627; yingst@psi.edu

15 July 2020

Also posted on the MEPAG web site, and may be referenced as follows:

MEPAG (2020), Mars as a Competitive Candidate for Inclusion in the New Frontiers Mission List: MEPAG Community Perspectives, R.A. Yingst, ed., 7 p. white paper posted July, 2020 by the Mars Exploration Program Analysis Group (MEPAG) at http://mepag.jpl.nasa.gov/decadal/index.html.
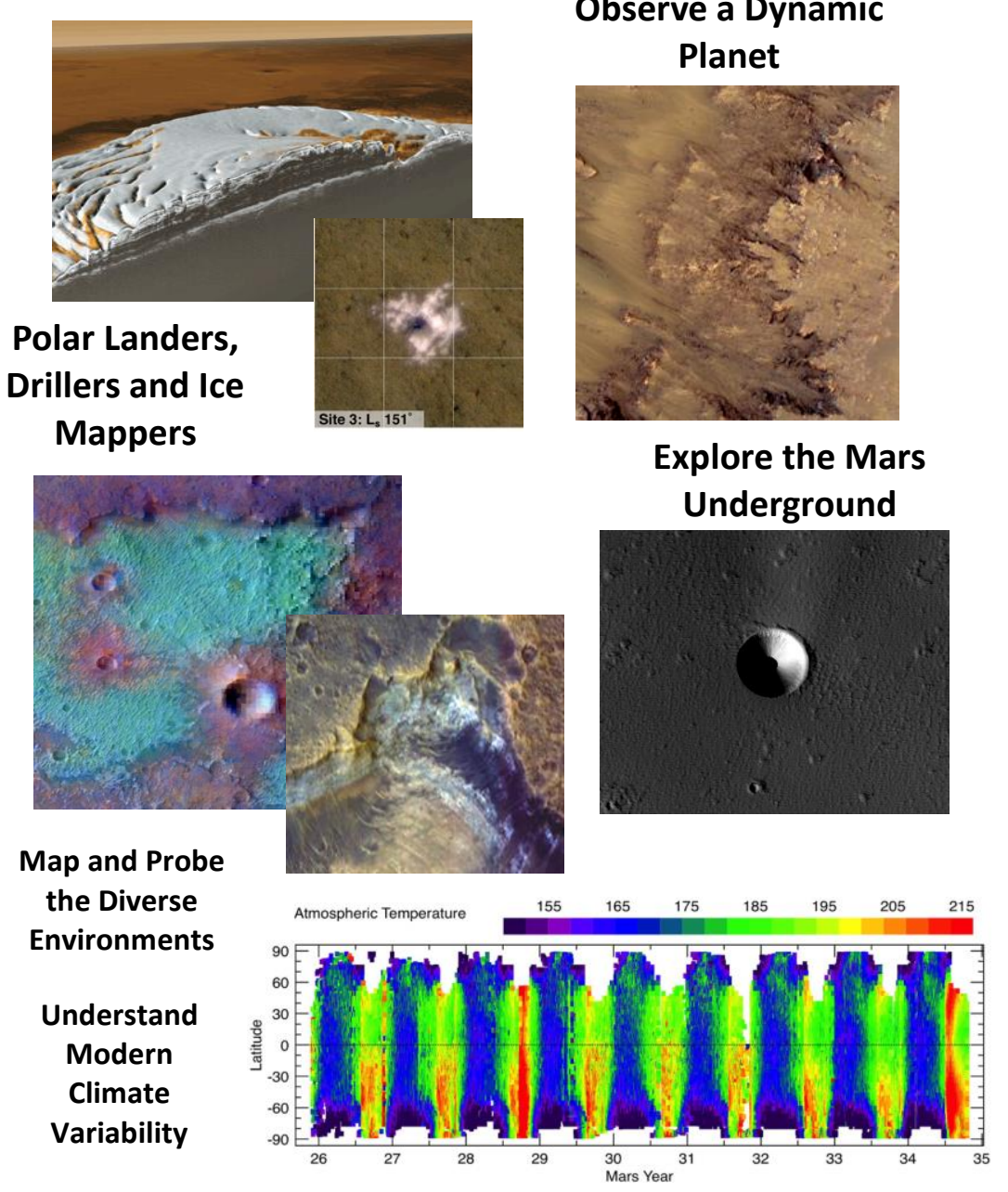

Observe a Dynamic

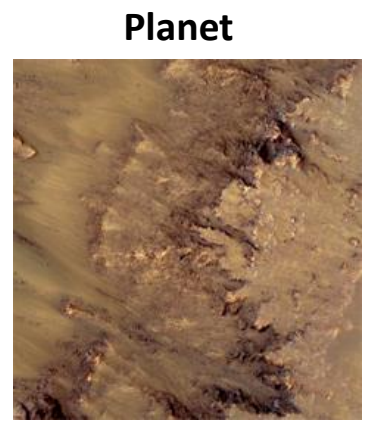

Explore the Mars 


\section{Introduction}

The Decadal Survey report is tasked [Survey] to "consider whether specific flight investigations with costs in the approximate range \$500-900 million (New Frontiers class) should continue to be specified or whether this mission class should be open in a manner similar to the Discovery program; if specific flight investigations are recommended, the report should provide a candidate list of objectives for each mission".

The Mars Exploration Program Analysis Group (MEPAG) Steering Committee submits this white paper as a statement of the consensus of the MEPAG community regarding the importance of including Mars in the list of candidate New Frontiers class missions.

\section{Should There Be a List?}

In August, 2019, the MEPAG community debated whether a curated list of allowable New Frontiers (NF) missions should exist [VM6 Finding]. Arguments for and against were discussed.

Three points were noted in support of an open competition (no list):

1. Open competition would enable response to rapidly changing priorities, whereas a curated list would not.

2. Making NF mission definition part of an open competition would remove concern about how to keep such a list up-to-date and fair to the entire community.

3. No solar system object would be excluded (e.g., there are currently no Mars or Mercury candidates).

Three factors were cited in support of a curated list of possible NF missions:

1. While it was agreed that open competition would allow rapid respond to science advancements, development of NF proposals require significant time and resources. As a result, a completely open call could overwhelm the supporting technical centers, leading to their internal processes and priorities (rather than the most compelling science) defining which proposals would go forward. Such key support centers could thus become de facto arbitrators and gatekeepers, as they would necessarily down-select proposals based on institutional concerns in addition to science-driven factors (such as internal perception of success or personnel/technical resource allocation). This would stifle both science-driven mission development and likely the diversity of mission types and mission teams.

2. It was agreed that a prioritized NF list would enable the Decadal Survey to set scientific priorities across the most expensive and technically challenging classes of missions (NF and Flagships), while retaining many advantages of open competition. There was concern that if NF was completely open (and thus decoupled from the Decadal Survey process), then the Decadal Survey vetting process would include only Flagships and constitute a much more limited assessment of science-driven mission activity.

3. It was noted that while no Mars candidate is in the present NF list, community discussions held during MEPAG meetings over the past several years and MEPAG Science Analysis Groups have demonstrated that there is high-priority Mars-related science that can be accomplished within the New Frontiers class, and that there are Mars missions which are competitive in this class. 
Ultimately, the arguments for a Decadal Survey-crafted and-curated, limited list of NF missions were seen as more compelling than arguments against.

Summary: MEPAG supports the current process in which the Planetary Decadal Survey creates a New Frontiers Candidate List.

\section{How Should that List be Updated beyond the Decadal Survey Process?}

MEPAG recognizes the occasional need for a course correction to the NF list and encourages that whatever process is used to alter the list between Decadal Surveys should be fair, science-driven, open, and clearly communicated to the community. In particular, better procedural definition of how to refresh the NF list between Decadal Surveys would strengthen confidence that the list represents the most pertinent New Frontiers-class science to be pursued by the community.

The mid-Decadal addition of Ocean Worlds to the NF list via the Committee on Astrobiology and Planetary Science (CAPS) raised concerns by many scientists. NF mission candidates were wellvetted by the Decadal Survey committee at great expense and effort, and it was felt that there should be a formal, compelling reason to change that list. However, the community recognized that such reasons can occur, due to new observational or analytical discoveries. Furthermore, while the list must reflect the science priorities of the community, NASA must retain the flexibility to implement that list based on opportunity, which sometimes requires dipping into lower priorities when the situation calls for it. While MEPAG does not endorse a specific plan for consideration of mid-Decadal changes to a NF mission candidate list, several suggestions were supported by the community:

- Prior to the mid-Decadal CAPS review, NASA could request that the appropriate Analysis Group assess the priority of the proposed mission candidate within their set of priorities. Alternately, NASA could ask all the Analysis Groups to comment, providing inputs to CAPS, on the priority of the new proposal.

- NASA could ask the NAS Midterm Decadal Review Committee to consider a proposed update, either driven by opportunity or as a matter of course. Alternately, a committee such as the New Opportunities in Solar System Exploration committee (NOSSE), which includes participation by a wide swath of the community through the Analysis Groups, could be tasked with reviewing and recommending any changes to the NF list.

We note that if the forthcoming Decadal Survey does not open up the NF list to Mars in general, or to Mars with focused science objectives (e.g., "Enceladus and Titan aimed at the search for life" in NF4), then the MEPAG community would recommend a formal process by which to request changes to the NF candidate list. This is needed in order for the community to be responsive to mid-Decadal discoveries achieved by ongoing NASA and international Mars missions and to keep pace with technological milestones that outpace the DS timeline.

Summary: MEPAG encourages a formal, science-driven method for editing a Decadal Surveyprovided NF mission candidate list, should mid-Decadal discoveries and technological advances warrant such a discussion. The inclusion of a well-defined process by which to request changes would be welcomed. 


\section{Candidate Science for the NF List}

The Mars exploration community has defined high-priority Mars and planetary science questions that can only be addressed via strategic use of NF-class missions to Mars. These should be considered for inclusion on a NF mission candidate list defined by the current Decadal Survey committee because NF provides unique and powerful opportunities for science and exploration that should be open to every community. High-priority science investigation areas that fall into the NF class include:

- In situ geochronology

- Regional/global-scale change detection from orbit

- Search for extant life (surface, ice caps, or deep subsurface)

- Recent Martian climate history (both orbital and surface missions)

- Geologic diversity/environmental transitions

Two recent MEPAG Science Analysis Group (SAG) studies addressed key elements of the above compelling science areas, identifying candidate missions in the New Frontiers class:

- $\quad$ The 2016 Next Mars Orbiter SAG [NEX-SAG] identified a NF-class orbiter that could pursue both science and resources (for humans on Mars) objectives. Key goals were mapping the surface and subsurface ice reservoirs and further characterizing from orbit the diverse mineralogy of the ancient surface, while providing new atmospheric measurements (water vapor profiles and horizontal winds). The MOIRE and MOSAIC Planetary Mission Concept Study (PMCS) investigations are exploring these goals in further detail.

- $\quad$ The 2019 Ice and Climate Evolution SAG [ICE-SAG] studied in greater detail what would be required to understand the processes of ice emplacement and removal. Several mission concepts were identified, including NEX-SAG-like orbiters, but also landers probing the subsurface layering while recording the surface environment. Some of these would have to operate through the challenge of the polar night environment.

Clearly, the MEPAG studies indicate that one area of compelling science is the cryosphere of Mars, both for itself and as a record of geologically recent climate change. White papers will introduce both of these studies to the Decadal Survey (Campbell and Zurek, 2020; Diniega and Putzig, 2020).

Below are several mission concepts based on recent NASA- or MEPAG-sponsored reports, as well as funded through PMCS. While these do not represent a prioritized list relative to any other NFclass science and mission concepts, and MEPAG does not offer endorsement, we provide this list to demonstrate the maturity of several potential mission concepts that would address NF-class, Mars-related science. Ongoing studies include:

- In situ geochronology is a non-Mars specific concept gaining significant traction in multiple communities for its ability to address key fundamental questions across the solar system. One potential set of missions is reported in the PMCS study on In Situ Geochronology for the Next Decade (PI: B. Cohen; Geochronology for the Next Decade). 
- PMCS for Mars NF-class mission concepts include Mars Orbiter for Resources, Ice and Environment (MORIE; PI: W. Calvin; PMCS-MORIE) and Mars Orbiters for Surface-Atmosphere-Ionosphere Connections (MOSAIC; PI: R. Lillis; PMCSMOSAIC).

- MORIE addresses high priority questions related to shallow subsurface ice, polar layer stratigraphy, and mineralogy of ancient diverse habitable environments. Its observations would address both resource and ice objectives (see NEX-SAG report).

- MOSAIC seeks to observe simultaneously the connections between ionosphere, atmosphere, surface and subsurface processes. This flotilla of spacecraft would follow water from surface and subsurface ice reservoirs through the middle and upper atmospheres, including loss to space. Although the constellation proposed by MOSAIC is well into the flagship class as a whole, there are New Frontiers and Discovery-class components of that constellation that could be provided by a consortium of international partners and/or be built up over time (e.g., MIC-SAG).

Other studies that have been proposed or investigated include:

- C. Stoker's Search for Modern Life on Mars would explicitly add Mars to the NF candidates search for extant life.

- V. Stamenovic's Deep Trek: Technology \& Mission Concepts would explore the subsurface habitability and life potential on Mars; this includes identification of NFclass concepts.

- L. Tamppari's Terrestrial Planets Comparative Climatology Mission Concept would measure atmospheric fields at both Mars and Venus with the same spacecraft and payload, using solar electric propulsion to navigate into and out of the planetary orbits.

- Several efforts have been directed at finding a cost-efficient way of putting multiple mobile platforms or stationary networks onto the surface of Mars, the purpose being to efficiently address multiple, interwoven, interdisciplinary questions that inform solarsystem-wide questions. Because of their numbers, several of these concepts might fall under NF.

There are many other concepts; the Mars Architecture Study Working Group received over 50 one-page concepts for possible instruments and missions to study various aspects of Mars. Most of these will be described in companion white papers for the Survey to consider.

Summary: MEPAG believes that there are several missions in the New Frontiers class needed to pursue science objectives that are compelling if we are to understand Mars as a system, and to address fundamental questions of habitability and climate change on terrestrial planets. The priority of the science investigations and goals is such is that these missions would be highly competitive with other areas of solar system exploration. Mars is still the "nearest habitable world" to Earth and the Martian climate still is most similar to our own.

\section{Relation to the Future Mars Exploration Program}

Many of the missions described above were included in one or more of the four "mission arcs," or interrelated, synergistic missions addressing high-priority science, that the Mars Architecture 
Strategy Working Group (MASWG) used as examples to demonstrate how a Mars Exploration Program, operating alongside the Mars Sample Return campaign, could pursue compelling science objectives by utilizing a suite of missions. While the ongoing development of small spacecraft capabilities could revolutionize exploration of nearby planets, there will always be some objectives that are still likely to require more capable spacecraft.

MASWG noted that [MASWG Preliminary Report]: "While individual missions in a mission arc could be achieved through the Discovery/New Frontiers competitive process, inclusion in a strategic program line would ensure a consistent approach with missions building on one another. [However] New Frontiers should not be closed to Mars missions if missions of that class were prohibited by inadequate funding of the Mars program."

Summary: Though MASWG has recommended NF-class science be funded through a dedicated Mars Exploration Program, advocating for a News Frontiers Mars candidate in the competed program is a judicious approach to pursue simultaneously at this time given the imminent discussion of the 2023-2032 Decadal Survey. Adjustments to such an approach could be considered pending the establishment of more specific details regarding the direction of the Mars Exploration Program.

\section{References:}

Campbell, B. and R. Zurek (2020), Mars Next Orbiter Science Analysis Group (NEX-SAG) White Paper for the 2023-2032 Decadal Survey. Posted at: https://mepag.jpl.nasa.gov/reports.cfm?expand=decadal

Diniega, S., N. E. Putzig + 20 co-authors (2020), White Paper Summary of the Final Report from the Ice and Climate Evolution Science Analysis group (ICE-SAG). White Paper for the 2023-2032 Decadal Survey. Posted at: https://mepag.jpl.nasa.gov/reports.cfm?expand=decadal 